\title{
Importance of reciprocal ST segment depression in leads V5 and V6 as an indicator of disease of the left anterior descending coronary artery in acute inferior wall myocardial infarction
}

Boris Strasberg, Avraham Pinchas, Gabriel I Barbash, Hanoch Hod, Shmuel Rat, Yedael Har-Zahav, Avraham Caspi, Samuel Sclarovsky, Jacob Agmon

\begin{abstract}
The purpose of this study was to determine the coronary angiographic correlations (specifically disease of the left anterior descending coronary artery) of reciprocal ST segment depression appearing during inferior acute myocardial infarction. Forty six patients (41 men and five women; mean age 56 years) were allocated into two groups based on the extent of precordial ST segment depression: widespread (V1-V6) ST depression $v$ localised (V1-V4) ST depression. Patients with no reciprocal ST depression or patients with ST depression in V1-V4 but with ST elevation in V5 and V6 (inferolateral acute myocardial infarction) were excluded. All patients were catheterised during hospital admission for infarction. Twenty four of the 28 patients with ST depression in V1-V6 had significant lesions in the left anterior descending coronary artery whereas 16 of the 18 patients with ST depression in V1-V4 had insignificant or no lesions in the left anterior descending artery. The sensitivity, specificity, and positive and negative predictive values of widespread ST depression in predicting disease in the left anterior descending coronary artery were $92 \%, 80 \%$, and $86 \%$ and $89 \%$ respectively.
\end{abstract}

In patients with inferior acute myocardial infarction and precordial ST depression, the extent of ST depression is of clinical significance. Widespread (V1-V6) ST depression suggests disease of the left anterior descending coronary artery, whereas localised ST depression (V1-V4) indicates its absence.

Inferior acute myocardial infarction in its early stages is usually accompanied by ST segment depression in the precordial leads. The clinical significance of this ST segment depression has been the subject of recent investigations because of the possibility that this reciprocal ST segment depression indicates additional anterior wall ischaemia. ${ }^{1}$ Furthermore, it has been suggested that ST segment depression is an indicator of disease of the left anterior descending coronary artery. $^{2}$ In this study, we evaluated the frequency of disease in the left anterior descending artery in patients with inferior acute myocardial infarction in relation to the extent (widespread $v$ localised) of precordial ST depression.

\section{Patients and methods}

We reviewed the admission electrocardiogram of patients admitted to hospital with a first inferior acute myocardial infarction and without previous acute myocardial infarction at any other site who underwent coronary angiography during the same hospital admission.

\section{PATIENTS}

We studied 46 patients with inferior acute myocardial infarction. Thirty six of these patients came from our patient population who had had coronary angiography during the same hospital stay, usually for clinically indicated reasons (post-infarction angina, young age) during the years 1986-87. Ten additional patients were selected from a cohort of 100 patients admitted with acute myocardial infarction and treated with thrombolytic agents, who had cardiac catheterisation performed within the next 48 hours. The total group consisted of 41 men and five women aged 39-73 (mean 56 years).

\section{SELECTION CRITERIA}

The diagnosis of acute myocardial infarction was based on the classic triad of severe chest pain, evolving ST-T changes with development of abnormal $Q$ waves, and an increase in the serum concentrations of cardiac enzymes. Patients were included if the admission electrocardiogram showed ST segment elevation of at least $1.5 \mathrm{~mm}$ in the inferior leads (usually leads III and aVF), and reciprocal ST segment depression defined as $>1 \mathrm{~mm}$ horizontal or downward sloping ST depression at $0.08 \mathrm{~s}$ from the J point of the QRS complex in the anteroseptal leads (usually V2 and V3). Patients with no ST segment depression in leads V1-V4 and/or patients with ST segment elevation ( $>1 \mathrm{~mm}$ ) in leads V5 and V6 (owing to lateral wall involvement) were excluded. The 46 patients included in the study were divided into two groups according to the extent of ST segment depression. The first group (28 patients) showed widespread ST segment depression (V1-V6) and the second 
Examples of electrocardiograms in patients with inferior acute myocardial infarction showing widespread V1-V6 (top panel) and localised V1-V4 (bottom panel) reciprocal $S T$ segment depression.

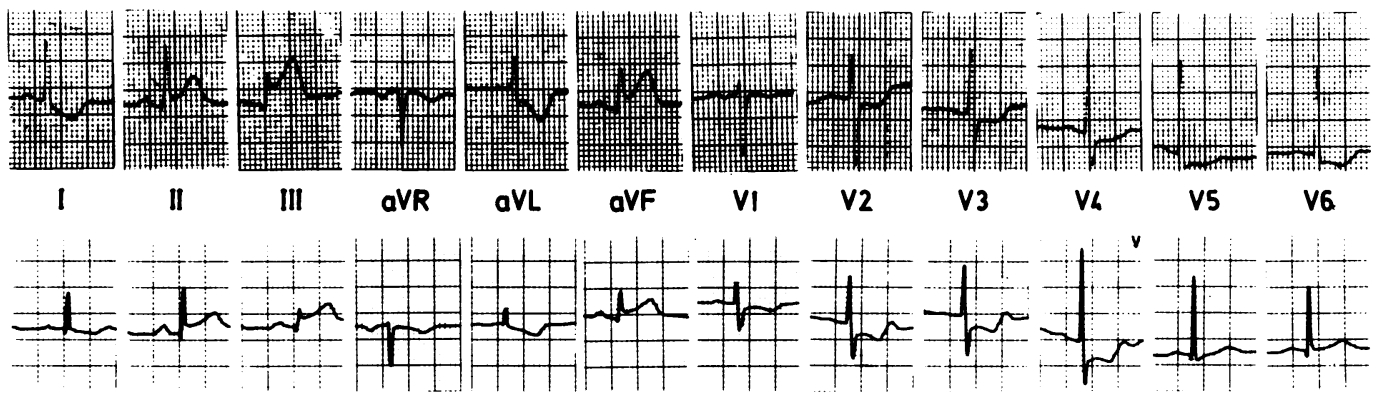

group (18 patients) showed ST depression only in V1 to V4 (isoelectric ST in V5 and V6) (figure).

Coronary angiography (by Judkins's or Sones's technique) was performed in all patients during the hospital admission for acute myocardial infarction. A significant coronary stenosis was defined as an obstruction of $\geqslant 70 \%$ of the luminal diameter in one or more projections. The coronary anatomy was reviewed by two observers who had no prior knowledge of the patient's electrocardiogram. For the purpose of this study, disease of the left main coronary artery (obstruction $>50 \%$ ) was regarded as disease of the left anterior descending coronary artery.

\section{STATISTICAI ANAIYSIS}

We used $\chi^{2}$ analysis (with Yates's correction) to determine whether the frequency of disease of the left anterior descending coronary artery was significantly different in the two electrocardiographic groups. In addition, we calculated the sensitivity, specificity, and positive and negative predictive value of widespread ST segment depression for disease of the left anterior descending coronary artery.

\section{Results}

Both groups were similar in terms of age, sex, and degree of ST depression in leads V1-V4. Of 28 patients with widespread ST segment depression (V1-V6) $24(86 \%)$ had significant lesions of the left anterior descending coronary artery (two patients had disease of the left main coronary artery) whereas 16 $(89 \%)$ of the 18 patients with ST segment depression in V1-V4 had insignificant or no disease of the left anterior descending coronary artery $(p<0.005)$. The sensitivity, specificity, and positive and negative predictive value of the presence of widespread ST segment depression for the recognition of disease of the left anterior descending coronary artery were $92 \%, 80 \%$, and $86 \%$ and $89 \%$ respectively.

\section{Discussion}

Much has been written about the significance and clinical implications of the presence or absence of ST segment depression in the precordial leads during inferior acute myocardial infarction. In general, it has been shown that precordial ST segment depression during inferior acute myocardial infarction indicates a larger infarction, predicting a worse prognosis, especially if the ST segment depression lasts

Though some studies showed that ST segment depression in patients with acute myocardial infarction indicates additional anterior wall ischaemia ${ }^{12-10}$ others showed that ST depression represents a reciprocal phenomenon that may be distorted by concomitant right ventricular or lateral left ventricular involvement. ${ }^{11-13}$

In several studies the coronary anatomy (especially that of the left anterior descending coronary artery) was examined in patients with inferior acute myocardial infarction with reciprocal ST segment depression and in those without. The results of these studies were also contradictory. Gibson et al and Lew et al found no difference in the frequency and the extent of coronary artery disease and in the frequency of disease of the left anterior descending coronary artery between patients with inferior acute myocardial infarction with precordial ST depression and in those without. ${ }^{56}$ In contrast, Gibelin et al found a significantly higher frequency of left coronary disease (left anterior descending and circumflex arteries) in patients with ST depression. ${ }^{14}$ Similarly, Salcedo et al found a high frequency of disease of the left anterior descending coronary artery $(96 \%)$ in patients with ST depression. ${ }^{2}$ None of the eight patients without reciprocal precordial ST depression had disease of the left anterior descending coronary artery. Similar results were reported by Akhras et al. ${ }^{10}$

We studied the frequency of disease of the left anterior descending coronary artery in patients with inferior acute myocardial infarction and precordial ST depression. We divided them into two groups based on the extent of ST depression (V1-V6 $v$ V1-V4 only). This division was arbitrary but was, however, based on our preliminary observations. We found confined to the anteroseptal leads (V1-V4) whereas direct anterior subendocardial ischaemia is usually seen in the lateral leads (V4-V6)..$^{15} 16$

We found that $24(86 \%)$ out of 28 patients with widespread ST depression had significant disease of the left anterior descending coronary artery, whereas only two $(11 \%)$ out of 18 patients with localised ST depression (VI-V4) did ( $p<0.005)$. These results gave sensitivity, specificity, and positive and negative predicfor more than 48 hours. $^{3-6}$ that pure reciprocal changes are usually 
tive values of $92 \%, 80 \%, 86 \%$ and $89 \%$ respectively.

Our results suggest that localised ST segment depression in V1-V4 is purely a reciprocal phenomenon, but that widespread ST depression (including V5 and V6) is a more complex phenomenon that probably indicates a reciprocal phenomenon and some additional ischaemic process unrelated to the acute occlusion of the artery, which is likely to be related to disease of the left anterior descending coronary artery. This additional ischaemic process is most probably subendocardial (represented by ST depression) and not necessarily severe enough to cause further haemodynamic embarrassment.

Our study had certain limitations. Firstly, we studied a specific subgroup of patients with inferior acute myocardial infarction. We included only patients with ST depression and patients without ST segment elevation in the lateral leads (V5 and V6); therefore we did not examine the implications of ST depression in V1-V4 in patients with inferolateral acute myocardial infarction. Secondly, since this is a retrospective study we cannot be certain that the precordial leads, especially V5 and V6, were properly positioned. If they were misplaced towards V4 the tracing could be misinterpreted as showing widespread ST depression. We excluded the only patient in whom there was a discrepancy between leads V5 and V6 (that is ST depression in V5 and none in V6).

In patients with inferior acute myocardial infarction and precordial ST depression the extent of precordial ST depression may be of clinical significance. Widespread (V1-V6) ST depression suggests multivessel disease (specifically of the left anterior descending coronary artery) whereas localised ST depression (V1-V4) indicates that there is no disease of the left anterior descending coronary artery.
1 Shah PK, Pichler M, Bermon DS, et al. Non-invasive identification of a high risk subset of patients with acute inferior

2 Salcedo JR, Baird MG, Chambers RJ, Beanlands DS. Significance of reciprocal ST segment depression in anterior precordial leads in acute inferior myocardial infarction: concomitant left anterior descending coronary artery disease? Am J Cardiol 1981;48:1003-8.

3 Gelman JS, Saltups A. Precordial ST segment depression in patients with inferior myocardial infarction: clinical implications. Br Heart $J$ 1982;48:560-5.

4 Ong L, Valdellon B, Coromilas J, Brody R, Reiser P, Morrison J. Precordial ST segment depression in inferio

5 Gibson RS, Crampton RS, Watson DD, et al. Precordial ST-segment depression during acute inferior myocardial infarction: clinical, scintigraphic and angiographic infarction: clinical, scintigraphic and

6 Lew A, Weiss T, Shah P, et al. Precordial ST segment depression during acute inferior myocardial infarction: early thallium-201 scintigraphic evidence of adjacent posterolateral or inferoseptal involvement. $\mathrm{J} \mathrm{Am} \mathrm{Coll}$ Cardiol 1985;5:203-9.

7 Billadello JJ, Smith JL, Ludbrook PA, et al. Implications of reciprocal ST segment depression associated with acute myocardial infarction identified by positron tomography $J$ Am Coll Cardiol 1983;2:616-24.

8 Goldberg HL, Borer JS, Jacobstein JG, Kluger J, Scheidt SS, Alonso DR. Anterior ST segment depression in acute inferior myocardial infarction: indicator of posterolateral infarction. Am J Cardiol 1981;48:1009-15.

9 Jennings K, Reid DS, Julian DG. Reciprocal depression of the ST segment in acute myocardial infarction. $\mathrm{Br} \mathrm{Med} \mathrm{J}$ 1983;287:634-7.

10 Akhras F, Upward J, Jackson G. Reciprocal change in ST segment in acute myocardial infarction: correlation with findings on exercise electrocardiography and coronary findings on exercise electrocardiography

11 Croft $\mathrm{CH}$, Woodward W, Nicod P, et al. Clinical implications of anterior ST segment depression in patients with acute inferior myocardial infarction. Am $J$ Cardio 1982;50:428-36.

12 Wasserman AG, Ross AM, Bogaty D, Richardson DW, Hutchinson RG, Rios JC. Anterior ST segment depression during acute inferior myocardial infarction: evidence for the reciprocal change theory. Am Heart 1983;106:516-20.

13 Lew AS, Maddahi J, Shah PK, et al. Factors that determine the direction and magnitude of precordial ST segment deviations during inferior wall acute myocardial infarction. Am J Cardiol 1985;55:883-8.

14 Gibelin P, Gilles B, Baudouy M, Guarino L, Morand P. Reciprocal ST segment changes in acute inferior myocardial infarction: clinical, haemodynamic and angiocardial infarction: clinical, haemodynamic and

15 Sclarovsky S, Davidson E, Lewin RF, Strasberg B, Arditti A, Agmon J. Unstable angina pectoris evolving to acute myocardial infarction. Am Heart $J$ 1986;112:459-62.

16 Sclarovsky S, Topaz O, Rechavia E, Strasberg B, Agmon J. Ischemic ST segment depression in $\mathrm{V}_{2}-\mathrm{V}_{3}$ on the presenting electrocardiographic feature of posterolateral wall myocardial infarction. Am Heart J 1987;113:1085-90. 